\title{
Novel Combinations for Wound Healing: Chitosan/PVA Nano Fiber; Royal Jelly, Probiotic
}

Saeed Ahmadi Majd*, Mohammad Rabbani Khorasgani and Firouzeh Khosravi

Department of Microbiology, University of Isfahan, Isfahan, Iran

*Corresponding author: Saeed Ahmadi Majd, Department of Microbiology, University of Isfahan, Isfahan, Iran, Tel: +98 313793 2288; E-mail: saeedmajd68@gmail.com

Received date: January 31, 2017; Accepted date: February 03, 2017; Published date: February 05, 2017

Copyright: (C) 2017 Majd SA, et al. This is an open-access article distributed under the terms of the Creative Commons Attribution License, which permits unrestricted use, distribution, and reproduction in any medium, provided the original author and source are credited.

\section{Nano Fiber}

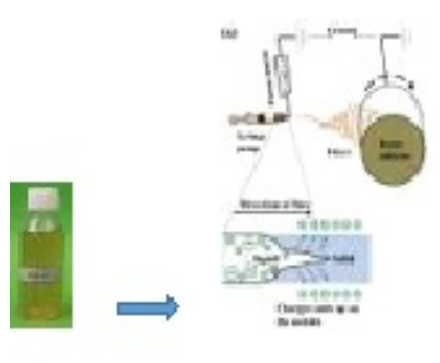

a

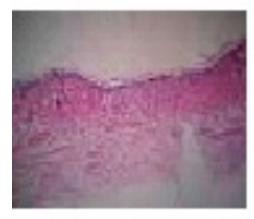

f

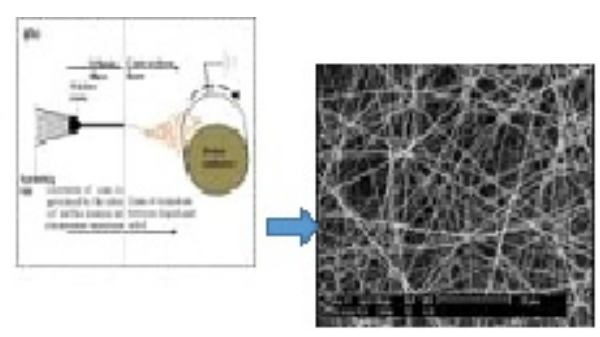

b

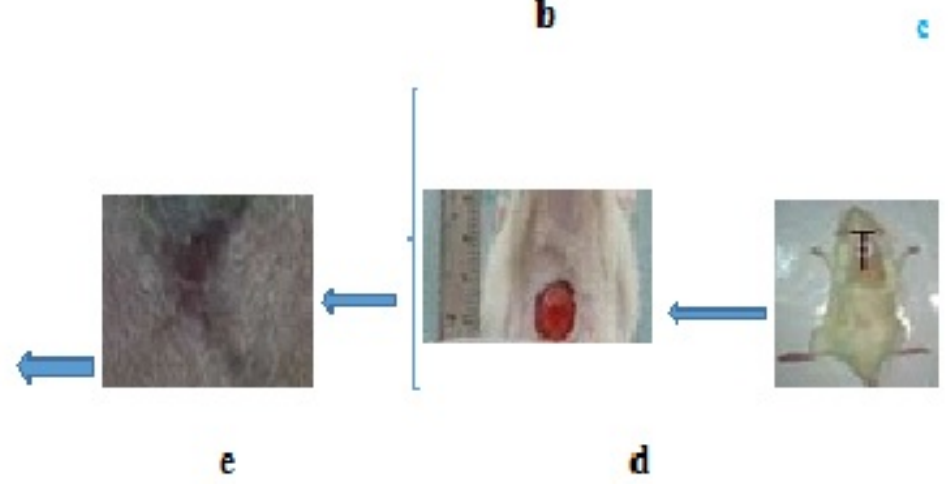

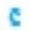
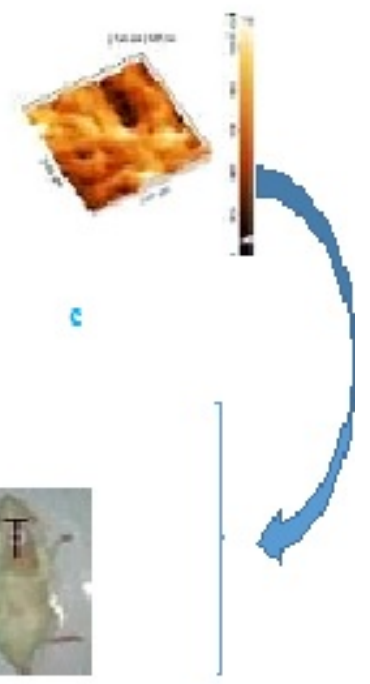

d

Figure 1: Schematic depicting, a: Chitosan solution, b: Electro spinning setup and phenomenon of electro spinning, c: electro spun fibers of Chitosan deposited on aluminum foil, d: Ulceration, e: Macroscopic evaluation f: Histological evaluation.

Nowadays, Diabetes is one of the most common diseases in the world. These diseases and disorders of the problems for the patient and the health system create. One of the long term complications of diabetes is neuropathy, or nerve problems which are involved in diabetic foot ulcers and prevalence of this disorder in patients between 12 and 50 percent mentioned. Diabetes mellitus is a group of metabolic diseases characterized by hyperglycemia resulting from defects in insulin secretion, insulin action, or both. Regarding the absence of sufficient efficacy of the available methods in diabetic wound healing, the use of alternative or complementary compounds has been considered (Figure 1 and 2).

Probiotics, chitosan and royal jelly are among these compounds. Currently, after creating experimental diabetes with streptozotocin in rats, effects of probiotics (Lactobacillus casei), exopolysaccharide of Lactobacillus casei, chitosan Nano fibers and N-chromosome royal jelly were investigated on wound healing of the mentioned animals and were compared to common medications in the market. In the experiment group with probiotics, the $24 \mathrm{~h}$ cultivation of Lactobacillus casei was used as a level having the most potential for producing exopolysaccharide. Macroscopic and histological findings indicate the reduction in the wound size and reduction in the epidermis and dermis length in the seventh and 14th days compared to the diabetic and healthy control groups. This issue indicates its positive effect on the process of wound healing. In the exopolysaccharide probiotic group, from the exopolysaccharide isolated from Lactobacillus casei, one percent solution was prepared and every other day, was used for the wound. The macroscopic and histological findings indicate its positive effects on the process of wound healing. In the chitosan Nano fibers groups, some fragments of the mentioned Nano fiber equal as $1.5^{\star} 1.5$ was put on the wound. The macroscopic and histological findings indicate the increase in the process of wound healing in this group in the seventh and 14th days. In the present study, undiluted and five percent $\mathrm{N}$-chromosome royal jelly were used which is a new combination and enjoys the feature of higher absorption than normal royal jelly. In undiluted $\mathrm{N}$-chromosome royal jelly, no improvement in the wound was obtained and even the wound got worsened, but in five 
Citation: Majd SA, Khorasgani MR, Khosravi F (2017) Novel Combinations for Wound Healing: Chitosan/PVA Nano Fiber; Royal Jelly, Probiotic. J Tissue Sci Eng 8: i101. doi:10.4172/2157-7552.1000i101

Page 2 of 2

percent $\mathrm{N}$-chromosome royal jelly, the wound size was reduced compared in the healthy and diabetic control groups. In general, the results obtained from the present study indicate a significant reduction in the wound size and facilitation in the process of wound healing of restorative materials used, with the exception of undiluted $\mathrm{N}$ chromosome royal jelly on the wounds in diabetic rats compared to diabetic and healthy control groups. Therefore, the application of the mentioned compounds to help diabetic wound healing can be investigated in further studies particularly in clinical trials in order that their application is considered after receiving legal permits (Figure 3).

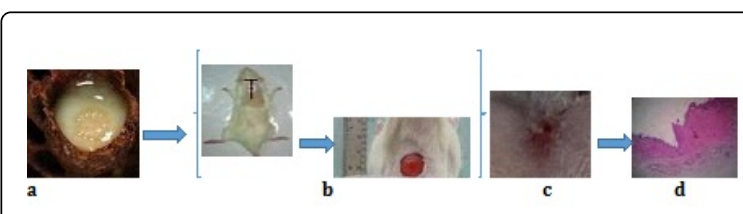

Figure 2: Schematic depicting, a: Royal Jelly, b: Ulceration, c: Macroscopic evaluation d: Histological evaluation.

\section{Probiotic}

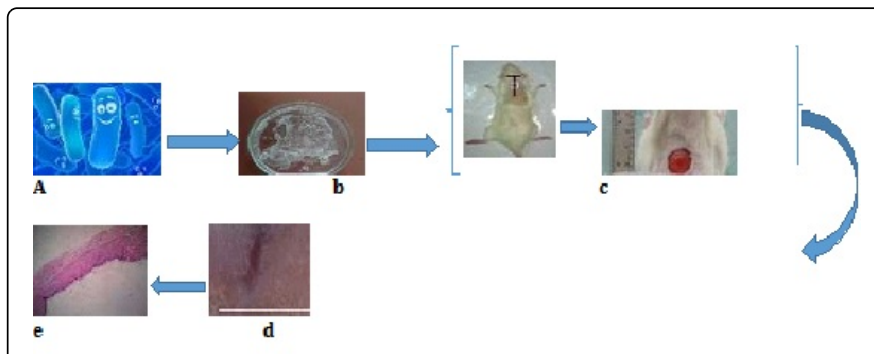

Figure 3: Schematic depicting, a: Probiotic, b: Exopolysaccharide, c: Ulceration, d: Macroscopic evaluation, e: Histological evaluation. 